\title{
A Preclinical Study of Cell-seeded Tubularized Scaffolds Specially Secreting LL37 for Reconstruction of Long Urethral Defects
}

\author{
YONGWEI LI ${ }^{1 *}$, JITAO WU $^{*}$, FAN FENG $^{1}$, CHANGPING MEN $^{1}$, DIANDONG YANG ${ }^{1}$, \\ ZHENLI GAO ${ }^{1}$, ZHE ZHU ${ }^{2}$, YUANSHAN CUI ${ }^{1}$ and HONGWEI ZHAO ${ }^{1}$ \\ ${ }^{1}$ Department of Urology, the Affiliated Yantai Yuhuangding Hospital of Qingdao University, Yantai, P.R. China; \\ ${ }^{2}$ Department of Stem Cell Biology and Regenerative Medicine, \\ Lerner Research Institute, Cleveland Clinic, Cleveland, OH, U.S.A.
}

\begin{abstract}
Aim: We constructed a new artificial, long tubular acellular matrix, seeded with autologous progenitor cells transfected with the sequence to produce the antibiotic peptide LL37 and another two common seeding cells, which might be adopted for patients requiring repair of long segment of the urethra. Materials and Methods: Autologous endothelial progenitor cells transfected by lentiviral vectors expressing antibiotic peptide LL37, as well as urothelial and smooth muscle cells from New Zealand white male rabbits, were cultured and seeded onto preconfigured acellular collagen-based tubular matrices ( $3 \mathrm{~cm}$ in length). Artificial conduits were created again in New Zealand white male rabbits and, then, evaluated by immunohistochemistry after 8 weeks. Results: Cell-seeded tubularized collagen scaffolds were found to be effective in repairing long urethral defects, whereas scaffolds without cells led to poor tissue development and structures. Conclusion: The artificial tissue engineered tubularized scaffolds combined with genetic methods resulted in vascularized autologous grafts, which may potentially be used for urethroplasty in patients requiring repair of a long segment of the urethra.
\end{abstract}

Urethral defect is a common disease caused by congenital hypospadias, complex urethral stricture and bladder/urethra

\footnotetext{
*These Authors contributed equally to this study.

Correspondence to: Hongwei Zhao, Department of Urology, the Affiliated Yantai Yuhuangding Hospital of Qingdao University, No. 20 East Yuhuangding Road, Yantai 264000, Shandong, P.R. China. Tel: +86 5356695579, Fax: +86 5356695579, e-mail: yddurology@163.com

Key Words: Urethral defect, acellular bladder matrix, endothelial progenitor cells, tissue engineering, LL37.
}

tumor invasion, etc. (1). Among most types of urethral injury, long tubular complexity urethral defect repair is the most challenging (2), especially for those with defective lengths exceeding $2 \mathrm{~cm}$ as they usually need tissue transplantation rather than just using the traditional repair operation that has a poor efficacy (3).

In recent years, acellular collagen matrix substitutes have become one of the most promising tools for the management of urethral defects (4-6). Acellular tissue matrices are usually prepared by manufacturing artificial scaffolds or by removing cellular components from tissues via mechanical and chemical manipulation to produce collagen-rich matrices (7). In the treatment of urethral defect, cells can be used for matrix-based tissue engineering strategies. Some articles have reported that LL37, an antibiotic peptide, has a broad spectrum effect (8). In addition, LL37 exerts different immunomodulatory functions, such as endotoxin-binding properties, modulation of pro-inflammatory response, chemotaxis, influence on cell proliferation and differentiation, promotion of wound healing and angiogenesis (9).

Therefore, the purpose of this study was to examine whether co-transplantation of bladder endothelial progenitor cells (EPCs) facilitating LL37 expression with genetic methods, urothelial cells (UCs) and smooth muscle cells (SMCs) in acellular collagen matrix was able to facilitate the reconstitution of the urethra and to achieve early and fundamental recovery of urethral defects.

\section{Materials and Methods}

Animal. The experimental procedures were approved by the Hospital's Institutional animal care and use committee. New Zealand white rabbits (male, 2.0-2.5 kg) were purchased from Beijing Wei-tong Li-hua Laboratory Animals and Technology Ltd, Beijing, China. 
Cell isolation and expansion. Cells were isolated and expanded according to previously published protocols $(10,11)$. Endothelial progenitor cells were separated from New Zealand white rabbits' femoral venous blood. The mucosal and muscular layers were mechanically separated. Muscle fragments were placed on culture dishes and muscle cells were expanded from these explants in M199 medium supplemented with $10 \%$ fetal bovine serum. The urothelial layer was mechanically separated from the bladder tissue using a scalpel and the cells were expanded in a similar manner in keratinocyte-serum-free medium (KSFM). Each cell culture required an average of five to six passages to reach appropriate numbers for seeding and implantation.

Cloning full length cDNAs of LL37 and construction of virus vector pGC FU LL37 GFP. The amplified polymerase chain reaction (PCR) products were cloned and sequenced. Based on the viral plasmid containing cDNA sequences of the antibacterial peptide gene $L L 37$, new primers were designed to amplify the full-length cDNA of $L L 37$ using high-fidelity Taq DNA polymerase (Toyobo, Osaka, Japan). The amplified PCR products and green fluorescent protein (GFP) were collectively cloned into the Lentivirus vector (Genechem, Montreal, QC, Canada) and then subjected to sequencing.

Acellular bladder matrix preparation and cell seeding. Rabbit bladder tissue was aseptically obtained from sacrificed animals. The submucosa was micro-dissected and isolated from the muscular and serosal layers. After removing all the cells through hypertonic saline+enzyme digestion+detergent washing methods, the tissue was freeze-dried into the membrane, sterilized with ultraviolet light for 24 hours before use and preserved at $4{ }^{\circ} \mathrm{C}$. UCs and SMCs were statically seeded on two surfaces of the scaffold in sequence using a glass pipette at a concentration of $4 \times 10^{6}$ cells per square centimeter. The cells were allowed to settle and attach to the scaffold in the incubator for $48 \mathrm{~h}$. The final seeding was performed with EPCs. These were statically seeded onto the sides of the scaffold and left again to settle for 48 hours prior to implantation. After the initial 4 days of dynamic cell seeding, the construct was placed in a 50/50 medium of Dulbecco's modified Eagle's medium (DMEM) and KSFM.

Western blot analysis. To investigate whether genetically modified EPCs secrete LL37 protein, EPCs were cultured in a 6well plate and transfected with pGC-FU-LL37-GFP or control plasmids for 3 days. The whole-cell lysate was then used to examine GFP and LL37 using western blot analysis, as described in previously published protocols (12). Glyceraldehyde 3phosphate dehydrogenase (GAPDH) was used as the internal control in each experiment.

Immunocytochemical and histological analysis. Five-micrometer sections of formalin-fixed paraffin-embedded tissues were processed and stained with hematoxylin and eosin (H\&E). Immunocytochemical analysis was performed using several specific antibodies. Broadly reacting monoclonal anti-pancytokeratins AE1/AE3 were used to identify the epithelial cell layers. Smooth muscle fibers were labeled with monoclonal alphasmooth muscle actin antibodies. Fluorescein isothiocyanate (FITC)CD34 monoclonal antibody was used to identify angiogenesis. FITC-lectin (FITC-UEA-1) and Dil-acetylated LDL (Dil-Ac-LDL) were used to identify EPCs. Immunolabeling was performed using the avidin-biotin detection system. Sections were counterstained with hematoxylin.

Surgical procedure. Ten New Zealand white male rabbits weighing 2.0-2.5 kg were anesthetized with $30 \mathrm{mg} / \mathrm{kg}$ pentobarbital sodium. The cell-seeded acellular collagen matrix expressed LL37 (4), no LL37 expressed (4) or only acellular collagen matrix (2) were trimmed and wrapped into the omentum of rabbits before using. The wound repair was performed using 6-0 Vicryl sutures in a continuous fashion. Gentle compression was applied to the surgical wound. Analgesia (buprenorphine, $0.01 \mathrm{mg} / \mathrm{kg}$ ) was administered intramuscularly at the end of the procedure followed by additional administrations every $8 \mathrm{~h}$ for the first 2 days. The rabbits were sacrificed at 8 weeks after surgery.

\section{Results}

Rabbit bladder EPCs, UCs and SMCs cultured in vitro; preparation of acellular bladder matrix scaffolds and toxicity tests. Successful cell cultures were obtained from every bladder biopsy used in the study. Bladder EPCs, UCs and SMCs were grown and expanded separately until sufficient amounts of cells were obtained. Bladder EPCs showed expression of Dil-Ac-LDL and FITC-UEA-1 (Figure 1A). Both the urothelial and smooth muscle cells showed expression of AE1/AE3 and a-SMA, respectively (Figure 1B).

A naturally derived acellular matrix tissue substitute was developed from a donor rabbit bladder. The processed scaffolds showed the presence of extracellular matrices (ECMs) with the absence of cells, as evidenced by histomorphological analysis. Both H\&E staining and scanning electron microscopy (SEM) showed no presence of cells after the decellularization process (Figure 1C). The decellularized scaffolds were assessed for toxicity to ensure the acellular nature of the matrix. The assessment showed that the relative growth rate of each treatment group exceeded $90 \%$ and the toxicity grade results were 0 or 1 (Figure 1D). Taken together, these results suggested that our prepared acellular collagen-based scaffolds were non-toxic and could, thus, be used for further experiments.

Cloning LL37 and induction of its expression in EPCs. EPCs (Figure 2A-a) were transiently transfected with pGC-FULL37-GFP lentiviral vector expression plasmid. Following transfection, we observed the intensive expression of GFP in EPCs through immunofluorescence assay (Figure 2A-b). Western blotting was used to detect the LL37 protein expression after EPCs were transfected with pGC-FU-LL37GFP; this showed that LL37 (47 kDa) was strongly expressed in EPCs (Figure 2B). The intensity of the LL37 signal (relative LL37 levels) in culture medium was quantified by densitometry, normalized by GAPDH level in cell lysates and, then, expressed as percentage of respective controls (transfected with empty lentiviral vector). 

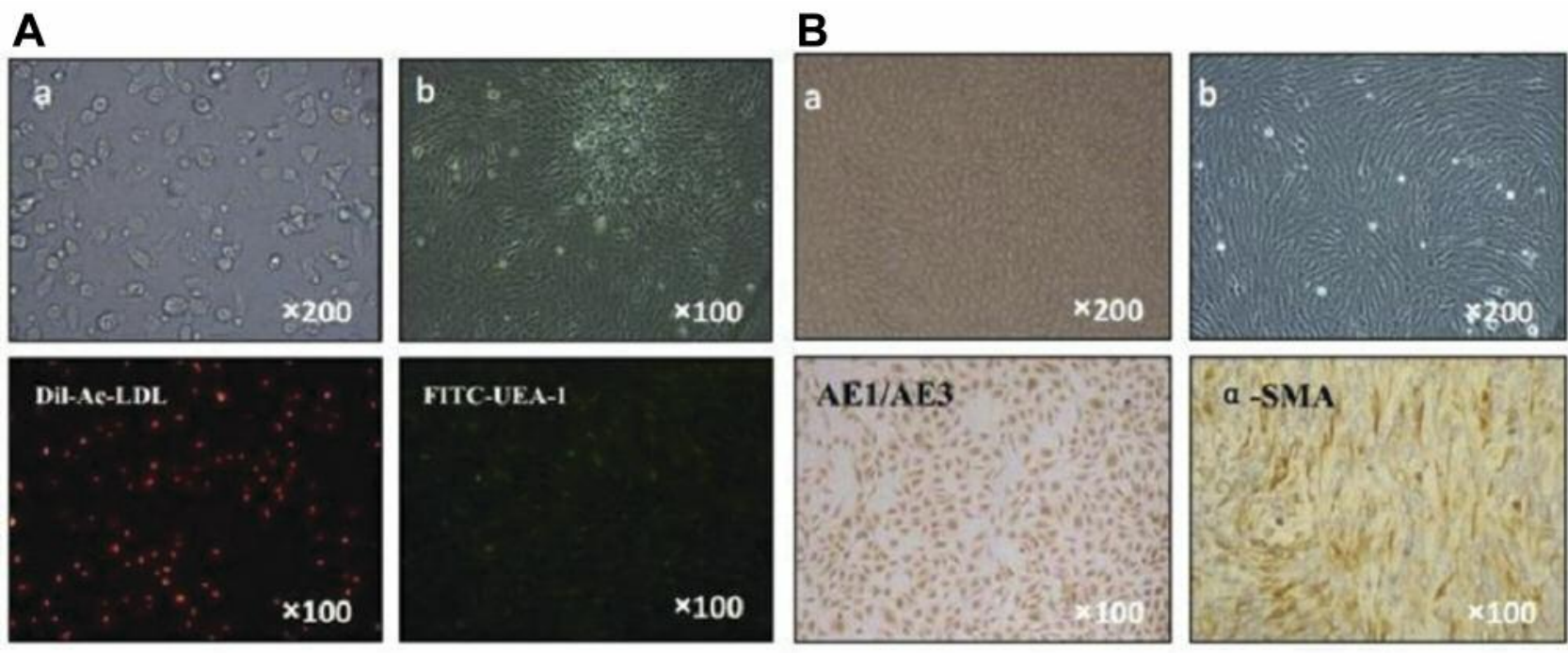

C

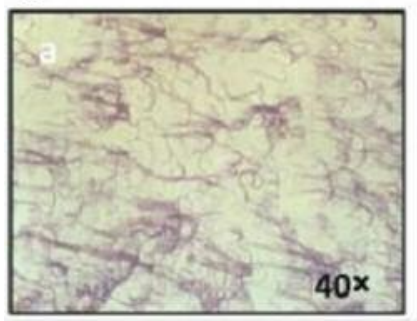

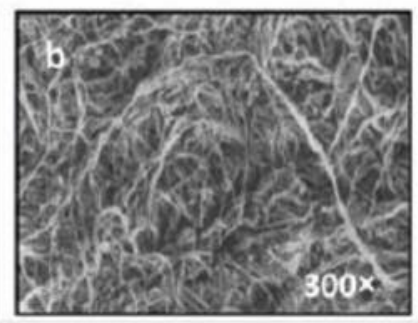

D

\begin{tabular}{|l|l|l|l|l|l|l|l|l|}
\hline \multirow{2}{*}{ Group } & \multicolumn{4}{|c|}{ RGR (\%) } & \multicolumn{4}{c|}{ Toxicity grade } \\
\cline { 2 - 10 } & 1 day & 4 days & 7 days & 10 days & 1 day & 4 days & 7 days & 10 days \\
\hline Positive control & 31.80 & 35.60 & 29.60 & 24.10 & 3 & 3 & 3 & 4 \\
\hline $100 \%$ & 97.90 & 96.80 & 91.20 & 95.40 & 1 & 1 & 1 & 1 \\
\hline $50 \%$ & 98.10 & 97.40 & 98.10 & 97.80 & 1 & 1 & 1 & 1 \\
\hline $25 \%$ & 101.00 & 98.50 & 97.80 & 97.50 & 0 & 1 & 1 & 1 \\
\hline $12.5 \%$ & 101.50 & 99.30 & 101.30 & 98.70 & 0 & 1 & 0 & 0 \\
\hline Black control & 100.00 & 100.00 & 100.00 & 100.00 & 0 & 0 & 0 & 0 \\
\hline
\end{tabular}

Figure 1. Three types of seeded cells were grown and expanded in culture and acellular bladder matrix scaffolds (extracellular matrices, ECMs) prepared and toxicity tests performed. (A) Bladder endothelial progenitor cells (EPCs), cultured 7 days (a) or 14 days (b). (B) Bladder urothelial cells (UCs) cultured 5 days (a) and smooth muscle cells (SMCs) cultured 7 days (b). EPCs show expression of Dil-Ac-LDL (red) and FITC-UEA-1 (green), UCs and SMCs show expression of AE1/AE3 (sandy) and a-SMA (claybank), respectively. (C) Collagen-based scaffolds derived from bladder were chemically treated to remove cells. Both hematoxylin and eosin (H\&E) staining (a) and scanning electron microscopy (SEM) (b) showed no presence of cells after the decellularization process. (D) Leaching solution toxicity test of collagen-based scaffolds. After EPCs cultured with different concentrations of ECMs leaching solution for 1, 4, 7 or 10 day(s) incubation, MTT assay was carried out at $540 \mathrm{~nm}$. Each data point represents the mean $\pm S E M$ of 3 replicates. Positive control, $1 \%$ phenol; Black control, endothelial cell growth medium-2 (EGM-2); RGR, relative growth rate.

Scaffolds seeded with EPCs, UCs and SMCs. In order to ensure collagen-based scaffolds had no toxic effects on seeded cells, prior to implantation we co-cultured EPCs, UCs and SMCs with collagen-based scaffolds, respectively. The growth curve of acellular bladder matrix co-cultured with UCs (Figure 3A-a), SMCs (Figure 3A-b) and EPCs (Figure $3 \mathrm{~A}-\mathrm{c}$ ) showed a good compatibility between the bladder cell matrix and the three cell types and minimal toxicity with little difference in growth observed between those cells cultured in the presence and those in the absence of the substrate. This result was further confirmed by the histologic analysis of ECM co-cultured with UCs (Figure 3B).

Surgical outcomes and histological analysis of reconstructed urethras. Experimental animals were sacrificed 8 weeks after implantation of cell-seeded matrix
(Figure 4A). The retrieved implants were analyzed grossly, histologically and using immunocytochemistry. Analysis showed formation of complete layers of transitional epithelium and well-developed smooth muscle. On the contrary, the group of cell-seeded matrix without LL37 expression showed inconspicuous vascularization and discontinuous epidermal growth.

Gross examination at retrieval showed normally appearing tissue without any evidence of fibrosis. The presence of a confluent transitional cell layer was confirmed by immunocytochemical analyses using pan-cytokeratin AE1/AE3 antibody (Figure 4B-a). Anti-alpha SMA antibody demonstrated the organized muscle fiber bundles and cord structure 8 weeks after implantation (Figure 4B-b). Histologically, the implanted matrices contained host cell infiltration and conspicuous angiogenesis by 8 weeks post- 
A
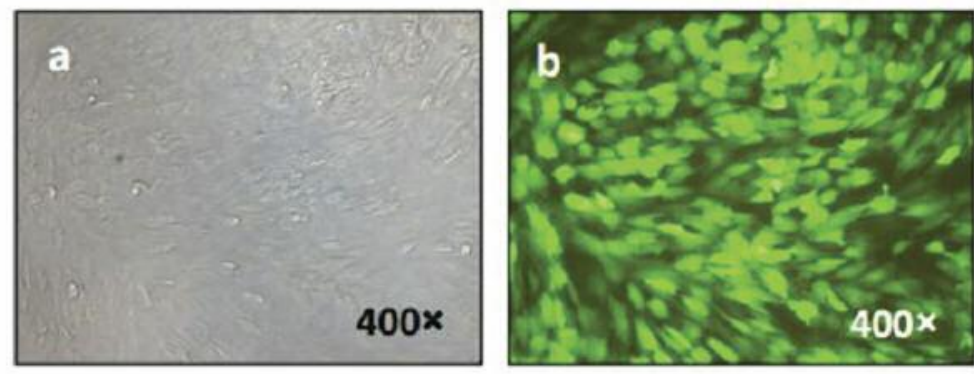

B

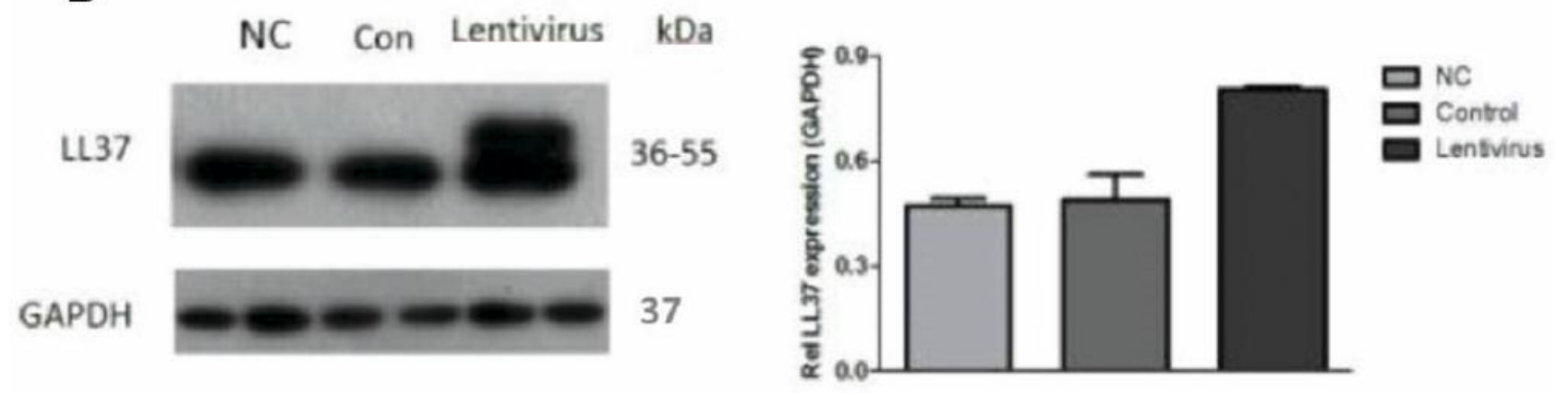

Figure 2. Induction of endothelial progenitor cells (EPCs) expressing LL37. (A) EPCs (a) were transiently transfected with pGC-FU-LL37-GFP lentiviral vector expression plasmid and an immunofluorescence assay was carried out (b). (B) Western blot analysis was used to detect the LL37 protein expression after EPCs had been transfected with pGC-FU-LL37-GFP. GAPDH was used as an internal control (reorganized the band - 3 to 3). The transfection of empty lentiviral vector construct into EPCs was used as an internal control (Con). Each data point represents the mean \pm SEM of 3 replicates. NC, Negative controls.

surgery (Figure 4B-c). Compared to the group with cellseeded matrix with LL37, non-LL37 groups showed no angiogenesis (Figure 4B-d).

\section{Discussion}

The urethra consists of the mucosa and submucosa and muscularis with epithelial cells and smooth muscle cells playing a key role, for instance, in resistance to the formation of urine calculi and urine erosion. Currently, the clinical efficacy for repairing complex urethral defects (where the defect length exceeds $2 \mathrm{~cm}$ ) is very poor, after traditional surgery. Raya-Rivera et al. reported for the first time that they used long tubular grafts created by tissue engineering in 5 patients with urethral strictures $(4-6 \mathrm{~cm})$ showing only one case of anastomotic stricture with good recovery after the treatment (13). Postoperative follow-up continued for 6 years at which point a biopsy confirmed that the urethral substitutes in all patients differentiated into normal urethral tissue. The concept of "tissue engineering" provides a new approach from the traditional therapy mode by sacrificing healthy tissue in order to repair damaged parts.
Many attempts have been made to identify alternative tissues generated by tissue engineering techniques that would serve as adequate urethral substitutes, with many studies showing positive treatment results (14). Tissue engineering techniques may be a suitable option for the creation of biocompatible urethral tissues. Cells can be derived from the heterologous (such as bovine, porcine), allogeneic (same species, different individual) or autologous tissue (15). Autologous cells derived from tissue biopsy are preferable in order to avoid the deleterious effects of immunosuppression (16). Following expansion in culture, attached to a support matrix, the cell-matrix construct is then implanted into the host. Acellular collagen matrices derived from the bladder have already been successfully applied both experimentally and clinically for urethral repair as an onlay graft (17). However, experimental data have shown that, in terms of tubularized urethral replacement, cell seeding is necessary to prevent stricture formation when matrices are used (13).

The innovation highlighted in this study is that the tissueengineered urethra created by the acellular bladder matrix, seeded with EPCs, UCs and SMCs that expressed LL37, which has antibiotic effect, is a useful preclinical research 

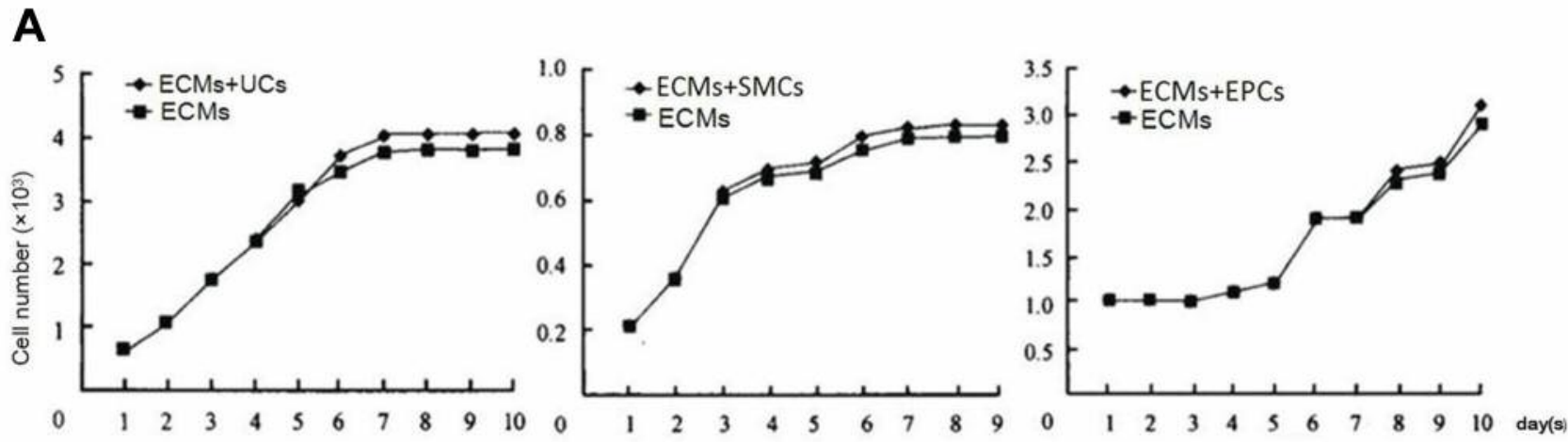

a

b

C

\section{B}

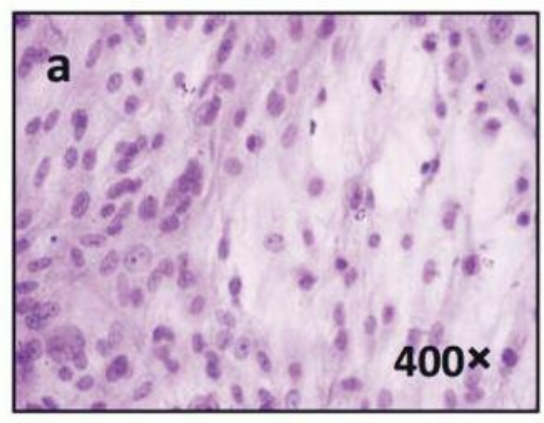

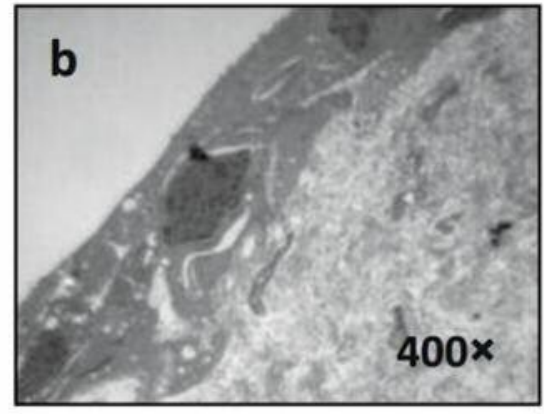

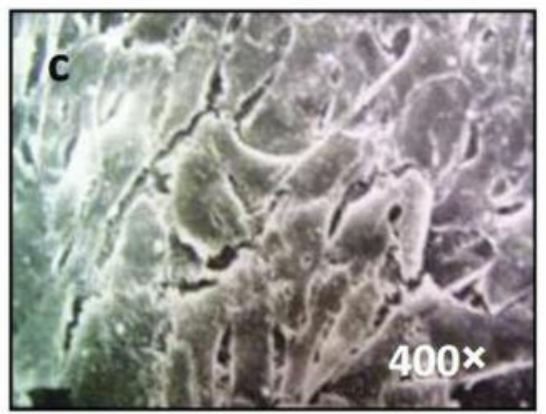

Figure 3. Toxicity detection and histological analysis of extracellular matrix (ECM) co-cultured with urothelial cells (UCs). (A) Growth curve of ECM co-cultured with UCs (a), smooth muscle cells (SMCs) (b) and endothelial progenitor cells (EPCs) (c), respectively. (B) Histological analysis of ECM co-cultured with UCs. Hematoxylin and eosin $(H \& E)$ staining $(a)$, transmission electron microscope (TEM) (b) and scanning electron microscopy $(S E M)(c)$.

tool for treating complex urethral defects. From a bladder biopsy, we were able to identify and isolate EPCs, UCs and SMCs by immunocytochemical or histological analysis and expand them until sufficient numbers were obtained for scaffold seeding. Meanwhile, we successfully transfected pGC-FU-LL37-GFP lentiviral vector expression plasmid into EPCs, with the cultured EPCs largely secreting LL37 protein. In this study we demonstrated that the urothelial cells formed a multilayered epithelial lining on the luminal side of the scaffold, while the muscle cells formed a corresponding layer on the outer surface with the endothelial progenitor cells cultivated on the urothelial cells.

To demonstrate that the seeded cells were able to contribute to tissue formation in vivo, firstly, we tested the toxicity of the acellular collagen matrix, based on its nontoxic characteristics and, secondly, we co-cultured three cell types with acellular collagen matrices. The results showed that the three types of cells that were seeded on the implanted scaffolds were able to survive and proliferate. In addition, we developed histological methods for analyzing the matrices co- cultured with UCs namely, H\&E staining combined with transmission electron microscopy (TEM) and SEM, which showed that the cells became integrated within the matrices.

Importantly, the surgical outcomes showed similar results to those reported in other studies $(11,13,18)$ that matrices seeded with three cell types have an improved phenotype compared to the control group in all histologic aspects. Both cell types were shown to contribute significantly to the multilayered tissue structure formed 8 weeks after implantation. Urothelial coverage and a complete muscle layer were seen in all implanted tissues with visible angiogenesis. In comparison, the control tissue showed contracture formation and invisible angiogenesis. We hypothesize that, when cell-seeded scaffolds are used, the rapid development of urothelial barrier along the luminal surface of the urethra may prevent urine leakage into the sub-urothelial tissue and associated fibrosis. Another contributing factor to the success of cell-seeded tubular urethral replacement could be the support provided by the rapidly developing muscle layer that keeps the urethra from 
A
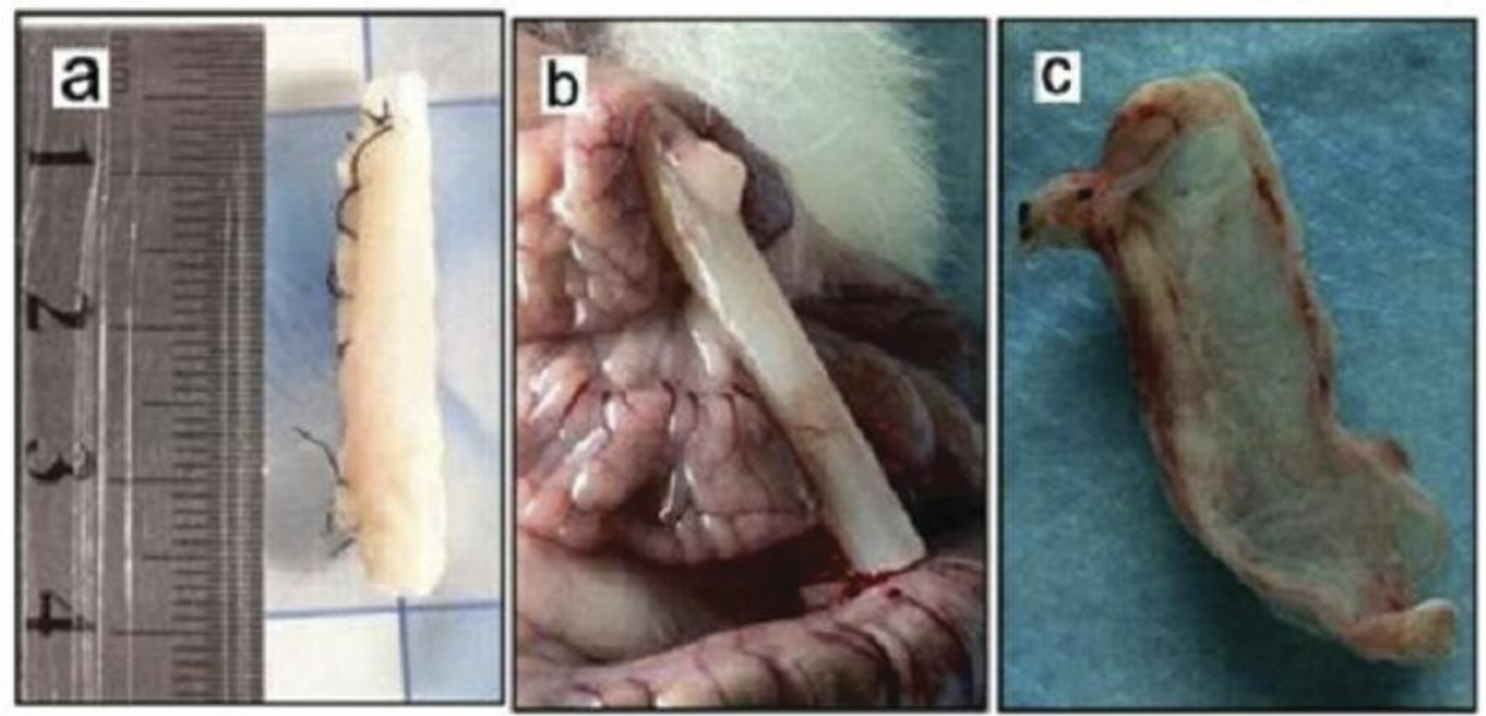

B

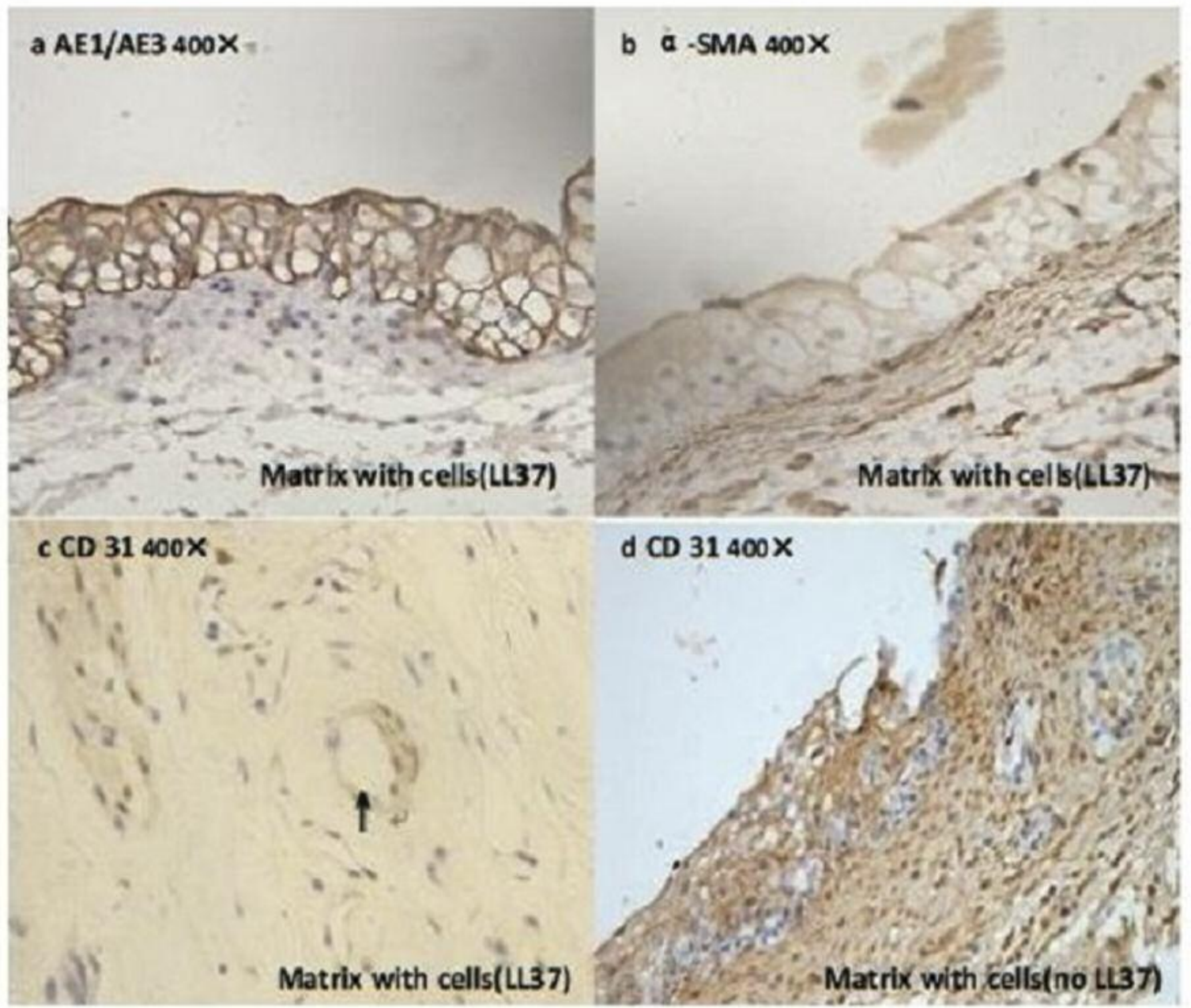

Figure 4. Surgical outcomes (A) and histological analysis of reconstructed urethras (B). (A-a) Tubularized urethral graft seeded with cells prior to surgery. (A-b) Implanted tubularized urethral graft at the time of surgery. (A-c) Implanted tubularized urethral graft at 8 weeks in rabbit. (B) Cellseeded matrix implanted in animals shows formation of complete layers of transitional epithelium and well-developed smooth muscle. On the contrary, the group of cell-seeded matrix without LL37 expression shows inconspicuous vascularization and discontinuous epidermal growth. 
collapsing and prevents wall adhesions. The massive secretion of LL37 in reconstruction tissue helped in avoiding infection and promoting angiogenesis. This mechanism would not be present in the unseeded scaffolds.

In conclusion, this work could open the way for the use of adequately long acellular bladder matrices seeded with autologous endothelial progenitor cells, especially combined with genetic methods, such as transfecting EPCs with lentiviral vectors expressing antibiotic peptide LL37, for the reconstruction of long urethral defects. Tissue-engineered urethras could be a new alternative source for reconstruction in the armamentarium of urologists. This study indicates that it is necessary to move toward clinical studies using this technique. More studies are required to establish the feasibility using the same concept for longer and more complicated defects.

\section{Disclosure}

There was no direct or indirect commercial incentive associated with publishing this article.

\section{Acknowledgements}

We thank our collaborators from central laboratory of Yantai Yuhuangding Hospital for providing technical support.

Funding

Supported by the National Nature Science Foundation of China (No. 81500587; No. 81572835), and by the Nature Science Foundation of Shandong Province (ZR2013HL070).

\section{References}

1 Onol SY, Onol FF, Gumus E, Topaktas R and Erdem MR: Reconstruction of distal urethral strictures confined to the glans with circular buccal mucosa graft. Urology 79: 1158-1162, 2012.

2 Gardikis S, Kambouri K, Giatromanolaki A, Agelidou M, Kalaitzis C, Giannakopoulos S, Touloupidis S and Vaos G: The use of a perimeatal-based penile skin flap to cover the repair of a tubularized incised plate urethroplasty: From experimental surgery to clinical practice. J Pediatr Urol 10: 469-473, 2014.

3 Koraitim MM: Transpubic urethroplasty revisited: total, superior, or inferior pubectomy? Urology 75: 691-694, 2010.

4 Eassa W, Jednak R, Capolicchio JP, Brzezinski A and ElSherbiny M: Risk factors for re-operation following tubularized incised plate urethroplasty: A comprehensive analysis. Urology 77: 716-720, 2011.

5 McAninch JW: Urethral reconstruction: a continuing challenge. J Urol 173: 7, 2005.
$6 \mathrm{Li} \mathrm{C}, \mathrm{Xu}$ YM, Liu ZS and Li HB: Urethral reconstruction with tissue engineering and RNA interference techniques in rabbits. Urology 81: 1075-1080, 2013.

7 Atala A: Tissue engineering of reproductive tissues and organs. Fertil Steril 98: 21-29, 2012.

8 Kittaka M, Shiba H, Kajiya M, Ouhara K, Takeda K, Kanbara $\mathrm{K}$, Fujita T, Kawaguchi $\mathrm{H}$, Komatsuzawa $\mathrm{H}$ and Kurihara $\mathrm{H}$ : Antimicrobial peptide LL37 promotes vascular endothelial growth factor-An expression in human periodontal ligament cells. J Periodontal Res 48: 228-234, 2013.

9 Li N, Yamasaki K, Saito R, Fukushi-Takahashi S, ShimadaOmori R, Asano M and Aiba S: Alarmin function of cathelicidin antimicrobial peptide LL37 through IL-36gamma induction in human epidermal keratinocytes. J Immunol 193: 5140-5148, 2014.

10 Chen F, Yoo JJ and Atala A: Acellular collagen matrix as a possible "off the shelf" biomaterial for urethral repair. Urology 54: 407-410, 1999.

11 Orabi H, AbouShwareb T, Zhang Y, Yoo JJ and Atala A: Cellseeded tubularized scaffolds for reconstruction of long urethral defects: A preclinical study. Eur Urol 63: 531-538, 2013.

12 De Filippo RE, Kornitzer BS, Yoo JJ and Atala A: Penile urethra replacement with autologous cell-seeded tubularized collagen matrices. J Tissue Eng Regen Med 9: 257-264, 2015.

13 Raya-Rivera A, Esquiliano DR, Yoo JJ, Lopez-Bayghen E, Soker $\mathrm{S}$ and Atala A: Tissue-engineered autologous urethras for patients who need reconstruction: An observational study. Lancet 377: 1175-1182, 2011.

$14 \mathrm{Fu}$ Q and Cao YL: Tissue engineering and stem cell application of urethroplasty: From bench to bedside. Urology 79: 246-253, 2012.

15 Olson JL, Atala A and Yoo JJ: Tissue engineering: Current strategies and future directions. Chonnam Med J 47: 1-13, 2011.

16 Li H, Xu Y, Xie H, Li C, Song L, Feng C, Zhang Q, Xie M, Wang $\mathrm{Y}$ and Lv X: Epithelial-differentiated adipose-derived stem cells seeded bladder acellular matrix grafts for urethral reconstruction: an animal model. Tissue Eng Part A 20: 774-784, 2014.

17 Atala A: Bioengineered tissues for urogenital repair in children. Pediatr Res 63: 569-575, 2008.

18 Orabi H, Bouhout S, Morissette A, Rousseau A, Chabaud S and Bolduc S: Tissue engineering of urinary bladder and urethra: Advances from bench to patients. Scientific World Journal 2013: $154564,2013$.
Received May 2, 2017

Revised May 25, 2017

Accepted May 26, 2017 